\title{
Revision and Return of a Coccidioidal Skin Test Reagent
}

\author{
Demosthenes Pappagianis - Suzanne M. Johnson
}

Published online: 11 October 2012

(C) Springer Science+Business Media B.V. 2012

The revival of a skin test reagent reported by Johnson et al. [1] provides the potential for renewal of a modality that is useful for evaluation of the immunologic response of patients with coccidioidomycosis. Ampel and Hector [2] in a prior commentary pointed out the value of skin testing and in vitro measurement of cellular immunity in coccidioidomycosis.

Even before the differing geographic distribution of the presumed two species of Coccidioides immitis and Coccidioides posadasii was demonstrated, the reactivity to coccidioidin (mycelial) or spherulin derived from the spherule-endospore (S/E) phase was demonstrated across broad geographic areas that were home to Coccidioides species.

While various coccidioidins had been prepared from mycelial phase cultures (reviewed by Smith et al. [3]), the most extensive evaluation and practical application were culture filtrates of Coccidioides grown in the synthetic (non-antigenic) modified asparagine-containing Bureau of Animal Industry medium previously used for tuberculin. Smith et al. [3] responding to the possible though unlikely antigenic differences among strains of Coccidioides, cultured multiple strains from California, Arizona, Texas and elsewhere. Grown static, the culture-yielded filtrates are harvested several weeks later, filter sterilized

D. Pappagianis $(\bowtie) \cdot S$. M. Johnson

Department of Medical Microbiology and Immunology,

School of Medicine, University of California, Davis,

CA 95616, USA

e-mail: a10pap@sbc.net and preserved with thimerosal 1:10,000 final concentration. From examination of the cultures, it was evident that autolysis of mycelium had taken place. Later, deliberate autolysis of mycelia resulted in release of potent serologic and skin test antigens [4]. HB Levine et al. [5] had successfully adapted the method and synthetic medium of John Converse for cultivation of the S/E phase of Coccidioides. They deliberately allowed the S/E phase cells to autolyze in sterile water to yield spherule-derived coccidioidin which they termed "spherulins". While Smith's coccidioidin was derived from multiple strains of Coccidioides, Levine et al. [5] prepared spherulin from a single strain, either strain Silveira, later categorized as C. posadasii, or strain 46, a C. immitis. As later recognized for serologic reactivity [6], antigens prepared from either species suffice for skin testing regardless of the geographic provenance of patients. Moreover, within the coccidioidin and spherulin were more than 20 antigenic components, some of which were not common to both preparations [7]. Coccidioidal skin test antigens do not affect serologic reactivity as may accompany histoplasmin skin tests [8]. Levine et al. [9, 10] in a number of papers provided evidence that spherulin detected a higher proportion of reactors (was more "sensitive") than coccidioidin among various groups of humans in endemic areas. However, a dissenting report of Gifford and Cantanzaro [11] suggested that there was no significant difference in sensitivity of the two reagents among patients with various forms of coccidioidomycosis. Anergy to coccidioidal skin tests had been considered an unfavorable response in patients with coccidioidal 
disease and could trigger a more rigorous treatment of patients. Therefore, there was an impression that spherulin would yield a positive dermal response in some patients with severe disseminated coccidioidal disease who would be anergic to coccidioidin. Skin test reactive components of coccidioidin were remarkably heat stable [12]. Levine et al. [5] pointed out that a reduction in potency of spherulin by heating indicated a difference in components between coccidioidin and spherulin. The heat stability of the polysaccharide-containing antigen(s) responsible for reaction with coccidioidal (IgM) raises an interesting question as to whether some antigen(s) may be both seroreactive and capable of skin test reactivity.

Finally, the issue of allergenicity of thimerosal appeared to complicate the validity of skin reactions to coccidioidin. Although Hurwitz et al. in 1938 [12] prepared a coccidioidal reagent that was preserved with phenol for the first field trial (in 449 human) cases, Smith selected thimerosal final concentration $1: 10,000$ for the early coccidioidin preparation. (Interestingly, commercial histoplasmin skin test reagent had been preserved with phenol.)

As recognized earlier and pointed out by Ampel et al. [13] and Johnson et al. [1], there was sufficient evidence that allergy could be induced by thimerosal, especially upon repeated injection of coccidioidin preserved with the organic mercury-containing compound. In order to reduce this interfering reactogenicity, the spherulederived coccidioidin Spherusol ${ }^{\circledR}$ which had originally been preserved with thimerosal 1:10,000 was diluted with buffered saline containing $0.4 \%$ phenol resulting in a final concentration of 1:1,000,000 thimerosal. This appeared to reduce allergenicity as only 2 of 125 human subjects reacted to the very dilute thimerosal in Spherusol ${ }^{\circledR}$ with uncertainty as to whether this was due to hypersensitivity.

For the first time in 12 years, a satisfactory and safe coccidioidal skin test reagent Spherusol $^{\circledR}$ has emerged. As Smith [3] pointed out, full recovery from coccidioidomycosis can result in immunity to exogenous reinfection. Testing with the new spherulederived coccidioidin could have important uses:

Testing for detection of past coccidioidal infection, for example, in selecting non-reactive recipients for a coccidioidal vaccine.

Testing for recent coccidioidal infection (skin test conversion).
Testing for anergy in a patient with active coccidioidal disease.

Testing in anticipation of employment or deployment in an endemic coccidioidal area (military personnel, farm workers, construction workers, fire fighters, archeologists and prisoners in institutions in endemic areas).

The reassurance of those who are skin test positive or the awareness of the risk of infections in skin testnegative individuals can have important implications.

\section{References}

1. Johnson R, Kernerman SM, Sawtelle BG, Rastogi SC, Nielsen HS, Ampel NM. A reformulated spherule-derived coccidioidin (Spherusol) to detect delayed-type hypersensitivity in coccidioidomycosis. Mycopathologia. 2012. doi: 10.1007/s11046-012-9555-6.

2. Ampel NM, Hector RF. Measuring cellular immunity in coccidioidomycosis: the time is now. Mycopathologia. 2010; 169:425-6.

3. Smith CE, Whiting EG, Baker EE, Rosenberger HG, Beard RR, Saito MT. The use of coccidioidin. Am Rev Tuberc. 1948;57:330-60.

4. Pappagianis D, Smith CE, Kobayoshi GS, Saito MT. Studies of antigen from young mycelia of Coccidioides immitis. J Infect Dis. 1961;108:35-44.

5. Levine HB, Cobb JM, Scalarone GM. Spherule coccidioidin in delayed dermal sensitivity reactions of experimental animals. Sabouraudia. 1969;7:20-32.

6. Paggagianis D. Current status of serologic studies in coccidioidomycosis. Curr Fungal Infect Rep. 2007;1:129-34.

7. Huppert M, Adler JP, Rice EH, Sun SH. Common antigens among systemic disease fungi analyzed by two-dimensional immmunoelectrophoresis. Infect Immun. 1979;23:479-85.

8. Deresinski SC, Levine HB, Kelly PC, Creasman RJ, Stevens DA. Spherulin skin testing and histoplasmal and coccidioidal serology: lack of effect. Am Rev Respir Dis. 1977;1116: 1116-8.

9. Levine HB, Gonzalez-Ochoa A, Ten Eyck DR. Dermal sensitivity to Coccidioides immitis. A comparison of responses elicited in man by spherulin and coccidioidin. Am Rev Respir Dis. 1973;107:379-86.

10. Stevens DA, Levine HB, TenEyck DR. Dermal senstivity to different doses of spherulin and coccidioidin. Chest. 1974; 65:530-3.

11. Gifford A, Cantanzaro A. A comparison of coccidioidin and spherulin skin testing in the diagnosis of coccidioidomycosis. Am Rev Respir Dis. 1981;124:440-4.

12. Hurwitz S, Young JE, Eddie BU. Coccidioides immitis intradermal skin reaction: a preliminary report of 449 cases. Cal West Med. 1938;48:87-9.

13. Ampel NM, Hector RF, Lindan CP, Rutherford GW. An archived lot of coccidioidin induces specific coccidioidal delayed-type hypersensitivity and correlates with in vitro assays for coccidioidal cellular immune response. Mycopathologia. 2006;161:67-72. 